$9-1-1993$

\title{
The 2'-Phosphate of NADP Is Critical for Optimum Productive Binding to 6-Phosphogluconate Dehydrogenase from Candida Utilis
}

Anthony J. Berdis

Cleveland State University, A.BERDIS@csuohio.edu

Paul F. Cook

Texas College of Osteopathic Medicine

Follow this and additional works at: https://engagedscholarship.csuohio.edu/scichem_facpub

Part of the Biochemistry Commons, and the Chemistry Commons

How does access to this work benefit you? Let us know!

\section{Recommended Citation}

Berdis, Anthony J. and Cook, Paul F., "The 2'-Phosphate of NADP Is Critical for Optimum Productive Binding to 6-Phosphogluconate Dehydrogenase from Candida Utilis" (1993). Chemistry Faculty Publications. 220.

https://engagedscholarship.csuohio.edu/scichem_facpub/220

This Article is brought to you for free and open access by the Chemistry Department at EngagedScholarship@CSU. It has been accepted for inclusion in Chemistry Faculty Publications by an authorized administrator of EngagedScholarship@CSU. For more information, please contact library.es@csuohio.edu. 


\title{
The 2'-Phosphate of NADP Is Critical for Optimum Productive Binding to 6-Phosphogluconate Dehydrogenase from Candida utilis
}

\author{
Anthony J. Berdis and Paul F. Cook
}

6-Phosphogluconate dehydrogenase $(6-\mathrm{PGDH})^{3}$ catalyzes the reversible oxidative decarboxylation of 6 -phosphogluconate (6-PG) to yield ribulose 5-phosphate (Ru 5-P) and $\mathrm{CO}_{2}$ (Eq. [1]).

NADP +6 -phosphogluconate $\rightleftharpoons$

$$
\mathrm{CO}_{2}+\text { ribulose 5-phosphate + NADPH }
$$

The kinetic mechanism of 6-phosphogluconate dehydrogenase from Candida utilis has been shown to approximate a rapid equilibrium random kinetic mechanism with allowances for E:NADP:Ru 5-P and E:NADPH:6-PG deadend complexes (1). In addition, the $\mathrm{pH}$ dependence of kinetic parameters has suggested a general base-general acid mechanism in which the general base accepts a proton from the 3-hydroxyl of 6-PG concomitant with hydride transfer and the general acid donates a proton to $\mathrm{C}-1$ in the formation of Ru 5-P after decarboxylation of the 3keto-6-phosphogluconate intermediate (2). In the above studies, it was not clear what role the 2 '-phosphate of NADP played in binding since its ionization was not observed. As a result, studies were undertaken with analogs of NADP.

In this paper, we present evidence that the enzyme uses NAD very poorly as a substrate and exhibits a random kinetic mechanism with marked antagonism of the binding of NAD and 6-PG at pH values of 7 and below. Data further suggest that the 2 -phosphate is crucial for the binding of the dinucleotide substrate.

for convenience); $\alpha$-NADP, $\alpha$-nicotinamide adenine dinucleotide 2 -phosphate; thio-NADP, $\boldsymbol{\beta}$-thionicotinamide adenine dinucleotide 2'-phosphate; APADP, $\beta$-3-acetylpyridine adenine dinucleotide 2 '-phosphate; $\epsilon$-NADP, $\beta$-nicotinamide $1-N^{6}$-ethenoadenine dinucleotide $2^{\prime}$-phosphate; HX-NADP, $\beta$-nicotinamide hypoxanthine dinucleotide 2 '-phosphate; 3 '-NADP, $\beta$-nicotinamide adenine dinucleotide 3 '-phosphate; NAD, $\beta$-nicotinamide adenine dinucleotide; SDS, sodium dodecyl sulfate: Mes, 4-morpholinepropanesulfonic acid; Ches, 2-(cyclohexylamino)ethanesulfonic acid. conate; 6-PGDH, 6-phosphogluconate dehydrogenase; BME, $\beta$-mercaptoethanol; Ru 5-P, ribulose 5-phosphate; ATP, adenosine 5 '-triphosphate; ATP-ribose, monophosphoadenosine $5^{\prime}$-diphosphoribose; $\beta$-NADP, $\beta$ nicotinamide adenine dinucleotide 2 '-phosphate (the plus sign is omitted 


\section{MATERIALS AND METHODS}

Enzyme. Cytoplasmic 6-phosphogluconate dehydrogenase from Candida utilis was purchased from Sigma. The enzyme was homogeneous by the criterion of SDS-polyacrylamide gel electrophoresis and had a specific activity of $48 \mathrm{U} / \mathrm{mg}$ assayed in the direction of oxidative decarboxylation using $100 \mathrm{mM}$ Hepes, $\mathrm{pH} \mathrm{7,3} \mathrm{mM} \mathrm{6-PG,} \mathrm{and} 0.4 \mathrm{mM}$ NADP. The enzyme was stored at $-20^{\circ} \mathrm{C}$ in a storage buffer containing $20 \mathrm{mM}$ Hepes, pH 7, $1 \mathrm{~mm} \beta$-mercaptoethanol, and $20 \%$ glycerol.

Chemicals. NADP and NAD were from Boehringer-Mannheim. 6 Phosphogluconate, ribulose 5-phosphate, ATP-ribose, Hepes, thioNADP, APADP, $3^{\prime}$-NADP, 6 -NADP, HX-NADP, ATP, acetyl phosphate, hexokinase, and acetate kinase were from Sigma. Mes and Ches buffers were from Ultrol. 3- $d$-6-Phosphogluconate was prepared from 3- $d$-glucose obtained from Merck, Sharpe, and Dohme Isotopes. Briefly, 3- $d$-glucose $(100 \mathrm{mM})$ was enzymatically converted to 3 - $d$-glucose 6 phosphate using $100 \mathrm{U}$ hexokinase with $4 \mathrm{mM}$ ATP and $8 \mathrm{mM} \mathrm{MgCl}_{2}$ in a $50-\mathrm{ml}$ total volume. The ATP was recycled using $200 \mathrm{U}$ of acetate kinase and $200 \mathrm{mM}$ acetyl phosphate. After full conversion, typically greater than $12 \mathrm{~h}$, the enzymes were removed by ultracentrifugation using an Amicon apparatus with a YM10 semipermeable membrane. The resulting solution was then treated with acid-washed, heat-activated charcoal and Dowex-50W $\left(\mathrm{H}^{+}\right)$and heated for $1 \mathrm{~h}$ to remove the ATP. The purified 3-d-glucose 6-phosphate solution was then lyophilized. Various amounts of crystalline 3-d-glucose-6-phosphate were then brominated as described by Berdis and Cook (1) and concentrated by rotoevaporation to a desired volume. The solution was titrated to the appropriate $\mathrm{pH}$ and used without further treatment. Unlabeled 6-phosphogluconate was prepared in an identical manner except that unlabeled glucose was used as the starting material. Unlabeled glucose was used in the deuterium isotope effect studies so that any contaminants would be equally present in both labeled and unlabeled 6-PG. All other chemicals and reagents were obtained from commercial sources and were of the highest purity available.

Initial velocity studies. All assays were carried out using a Gilford 250 spectrophotometer equipped with a strip-chart recorder to measure the appearance of NAD (P)H, $\epsilon-\mathrm{NADPH}, 3^{\prime}-\mathrm{NADPH}$, and HX-NADPH at $340 \mathrm{~nm}$, thio-NADPH at $395 \mathrm{~nm}$, and APADPH at $363 \mathrm{~nm}$. Rates were calculated for thio-NADPH and APADPH using extinction coefficients of 11,300 and $9100 \mathrm{M}^{-1} \mathrm{~cm}^{-1}$, respectively. The temperature was maintained at $25^{\circ} \mathrm{C}$ using a circulating water bath to maintain constant temperature of the thermospacers of the cell compartment. Reaction cuvettes were $1 \mathrm{~cm}$ in path length and $1 \mathrm{ml}$ in volume. All cuvettes were incubated for at least $10 \mathrm{~min}$ in a water bath prior to the initiation of reaction. Initial velocity data using dinucleotide analogs were obtained under conditions in which the dinucleotide substrate was varied at several fixed levels of 6-PG.

$\mathrm{pH}$ studies. Initial velocity data were obtained as a function of $\mathrm{pH}$ under conditions in which NAD was varied at several fixed levels of 6 . PG. The pH was maintained using the following buffers at a final concentration of $100 \mathrm{mM}$ : Mes, 5.5-6.5; Hepes, 6.5-8.5; Ches, 8.5-10. Sufficient overlaps were obtained when buffers were changed to correct for spurious buffer/ionic strength effects; no effects were detected. The $\mathrm{pH}$ was measured before and after initial velocity data were recorded with no significant change detected.

Deuterium isotope effects. Primary deuterium isotope effects with 3 d-6-PG using NAD as the dinucleotide substrate were obtained as a function of $\mathrm{pH}$ by direct comparison of initial velocities. Initial velocity data, in most cases, were obtained by varying the concentration of one substrate at saturating concentrations $\left(>20 K_{m}\right)$ of the other. The concentrations of the 6-PG and the 3-d-6-PG stock solutions were determined enzymatically using 6-PGDH. Data were plotted in the form of Lineweaver-Burk plots, which were linear. Isotope effects were estimated by fitting the appropriate rate equations to the data.

NAD-NAD stacking. There was a possibility that NAD-NAD stacking occurred at the high concentrations of NAD used in the initial velocity studies. To test this possibility, the absorbance of NAD at 259 $\mathrm{nm}$ was measured as a function of its concentration. Spectrophotometer cells were $0.1 \mathrm{~mm}$ in path length. A plot of absorbance versus NAD (up to $10 \mathrm{~mm}$ ) is linear (data not shown), and the $\epsilon$ is constant (data not shown).

Product and dead-end inhibition. Inhibition patterns were obtained using Ru 5-P as an analog of 6-PG with NAD maintained at a nonsaturating concentration varying the concentration of 6-PG and with 6PG maintained at a nonsaturating concentration varying the concentration of NAD at several concentrations of $\mathrm{Ru} 5$-P, including zero, at pH 6, 7, and 8, respectively. Inhibition patterns were obtained using ATP-ribose as a dead-end analog of NAD with 6-PG maintained at a saturating concentration varying the concentration of NAD and with NAD maintained at a nonsaturating concentration varying the concentration of $6-\mathrm{PG}$ at several concentrations of ATP-ribose, including zero, at $\mathrm{pH} 6,7,8$ and 9 , respectively.

Data processing. Reciprocal initial velocities were plotted against reciprocal substrate concentrations. When possible, data were fitted using the appropriate rate equations and computer programs developed by Cleland (3). Data conforming to a general sequential mechanism were fitted using Eq. [2]. Data conforming to a series of parallel lines with competitive substrate inhibition were fitted using Eq. [3]. Kinetic parameters from all other initial velocity data were obtained by graphical analysis. Data for competitive inhibition were fitted using Eq. [4], and data for noncompetitive inhibition were fitted using Eq. [5].

$$
\begin{aligned}
& v=V \mathrm{AB} /\left(K_{\mathrm{ie}} K_{\mathrm{b}}+K_{\mathrm{a}} \mathrm{B}+K_{\mathrm{b}} \mathrm{A}+\mathrm{AB}\right) \\
& v \approx V \mathrm{AB} /\left[K_{\mathrm{a}} \mathrm{B}\left(1+\mathrm{B} / K_{i \mathrm{~B}}\right)+K_{\mathrm{b}} \mathrm{A}+\mathrm{AB}\right] \\
& v=V \mathrm{~A} /\left(K_{\mathrm{a}}\left[1+1 / K_{\mathrm{is}}\right]+\mathrm{A}\right) \\
& v=V \mathrm{~A} /\left(K_{\mathrm{a}}\left[1+\mathrm{I} / K_{\mathrm{is}}\right]+\mathrm{A}\left[1+K_{\mathrm{i}}\right]\right)
\end{aligned}
$$

In Eqs. [2-6], $v$ is the initial velocity, $V$ is the maximum velocity, A, B, and I are reactant and inhibitor concentrations, $K_{\mathrm{a}}$ and $K_{\mathrm{b}}$ are the $\mathrm{Mi}$ chaelis constants for $\mathrm{A}$ and $\mathrm{B}, K_{\mathrm{ia}}$ is the dissociation constant for $\mathrm{A}$, and $K_{\mathrm{IB}}$ is the substrate inhibition constant for B.

Data for deuterium isotope effects were fitted using Eq. [6]

$$
v=V \mathrm{~A} /\left[(K+\mathrm{A})\left(1+F_{\mathrm{i}} E_{v}\right)\right]
$$

where $F_{\mathrm{i}}$ is the fraction of deuterium label in the substrate $(99.7 \%)$ and $E_{v}$ is the isotope effect minus 1 assuming equal isotope effects on $V$ and $V / K$.

\section{RESULTS}

Dinucleotide substrate analogs. Initial velocity patterns were obtained under conditions in which the dinucleotide substrate analog was varied at different fixed levels of 6-PG in the direction of oxidative decarboxylation. Dinucleotide analog structures are provided in Fig. 1. The initial velocity patterns obtained using $\beta$-NADP, thio-NADP, $\epsilon-N A D P, H X-N A D P$, and APADP are symmetrical and intersect to the left of the vertical ordinate, suggesting a sequential mechanism. Initial velocity patterns using $3^{\prime}$-NADP as the dinucleotide were qualitatively similar to the pattern obtained using NAD (discussed below). The $V$ and $V / K_{3}$-NADP were obtained at a saturating concentration of $6-\mathrm{PG}$. Although $\alpha-\mathrm{NADP}$ is not a substrate its $K_{\mathrm{i}}$ as a competitive inhibitor versus NADP was obtained. A list of kinetic parameters for the dinucleotides is given in Table I. 


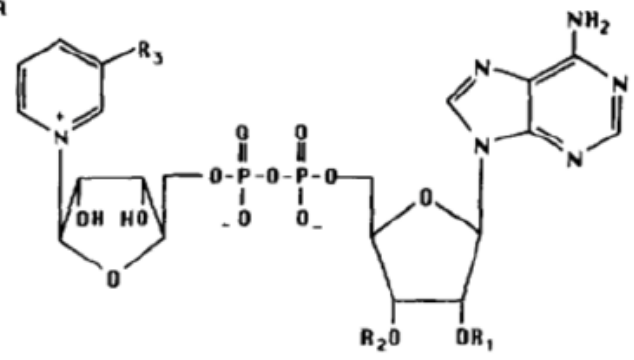

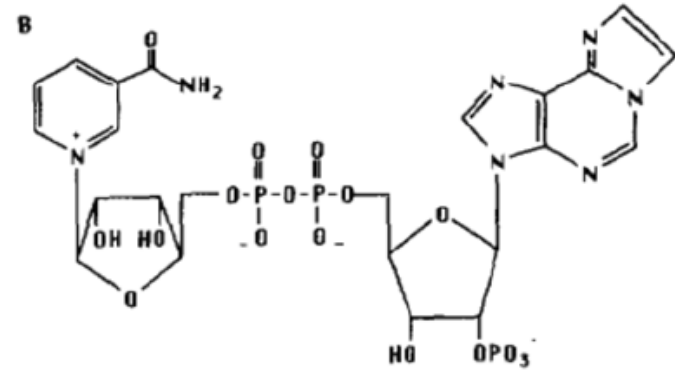

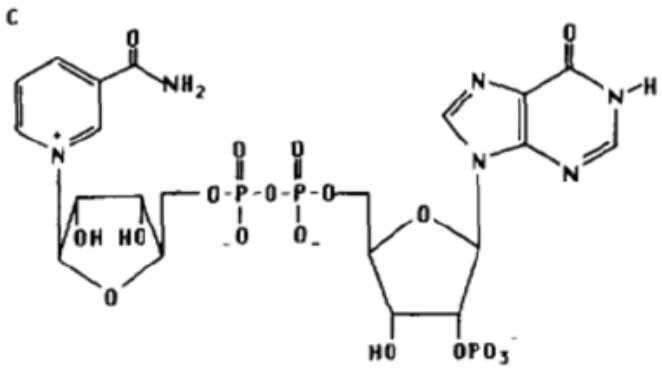

FIG. 1. Structures of the dinucleotide substrates. (A) Refer to Table IV for exact structures of $R_{1}$ and $R_{2}$ for each dinucleotide. (B) Structure of nicotinamide $1, N^{6}$-ethenoadenine dinucleotide phosphate $(\epsilon-N A D P)$. (C) Structure of HX-NADP.

Initial velocity patterns in the absence of inhibitors using $N A D$ as the dinucleotide substrate. Initial velocity patterns fall into two categories dependent on the $\mathrm{pH}$ at which data were collected. Data obtained below $\mathrm{pH} 8$ varying 6-PG at several fixed levels of NAD give a series of nearly parallel lines at low 6-PG with apparent substrate inhibition at all values of NAD, although the inhibition appears to be largely eliminated at $10 \mathrm{mM}$ NAD. Plotting the data varying NAD at several fixed levels of 6-PG results in a normal intersecting pattern at low 6PG concentrations with competitive substrate inhibition at higher concentrations of 6-PG. An example of this behavior obtained at $\mathrm{pH} 7$ is shown in Fig. 2. At pH 8 and above, intersecting initial velocity patterns are observed varying 6-PG at several fixed levels of NAD (or plotting the data the other way), indicative of a sequential mechanism. Phosphate and sulfate up to concentrations of 50 and $20 \mathrm{mM}$, respectively, had no stimulatory effect on the maximal velocity nor any effect on the Michaelis constant for NAD. Kinetic parameters are summarized in Table II.

Product and dead-end inhibition using NAD as the di-

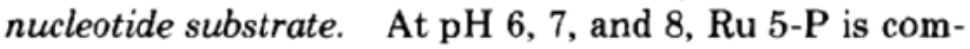

petitive vs 6-PG at nonsaturating NAD and noncompetitive vs NAD at nonsaturating 6-PG. ATP-ribose, a deadend analog of NAD $(P)$ is competitive vs NAD at saturating 6-PG at $\mathrm{pH}$ values of $6,7,8$, and 9 and noncompetitive versus 6-PG at nonsaturating $\mathrm{NAD}$ at $\mathrm{pH} 6,8$, and 9. At pH 7, inhibition by ATP-ribose vs 6-PG at nonsaturating NAD cannot be carried out since the lowest 6PG concentration that can effectively be used is saturating. There is, however, inhibition by ATP-ribose under these conditions, and thus inhibition is either noncompetitive or uncompetitive. Based on the inhibition pattern obtained at lower $\mathrm{pH}$, the former is most likely. Inhibition constants are summarized in Table III.

$p H$ dependence of kinetic parameters using NAD as the dinucleotide substrate. Because of the high $K_{m}$ for NAD, it is difficult to accurately measure the kinetic parameters at all $\mathrm{pH}$ values. From the data obtained, however, it appears that $V_{\max }$ is $\mathrm{pH}$ independent above $\mathrm{pH} 8$ and that the $V / K_{6 \cdot \mathrm{PG}}$ decreases on the basic side with a $\mathrm{pK}$ of about 8 (data not shown).

Deuterium isotope effects using NAD as the dinucleotide substrate. Deuterium isotope effects using NAD as the dinucleotide substrate for the 6-PGDH reaction were performed at $\mathrm{pH}$. The isotope effects on $V, V / K_{\mathrm{NAD}}$, and $V / K_{6 \cdot \mathrm{PG}}$ are equal with a value of $2.3 \pm 0.1$. This indicates that the kinetic mechanism of 6-PGDH using $\mathrm{NAD}$ as the dinucleotide substrate is rapid equilibrium random at $\mathrm{pH} 8$.

\section{DISCUSSION}

Interpretation of dinucleotide substrate analog studies. Initial velocity patterns in the absence of products were obtained for a number of dinucleotide analogs. All of the analogs tested with the exception of $\alpha$-NADP are substrates for 6-PGDH (Table I). $\alpha$-NADP is a competitive inhibitor. For most of the substrate analogs tested, initial velocity patterns intersect to the left of the ordinate, indicative of a sequential mechanism. Exceptions to this are NAD (Fig. 2) and 3'-NADP, which exhibit an apparently parallel initial velocity pattern. The reasons for this deviation from a sequential initial velocity pattern will be discussed below.

For all substrates (Table I) similar changes are observed in $V$ and $V / K_{6 \cdot P G}$ with any dinucleotide, as long as a 2'phosphate is present on the adenosine ribose (Fig. 2). Data obtained using alternative dinucleotide substrates suggest that the 2 -phosphate is crucial for the binding and the proper orientation of the dinucleotide substrate. Lower $V / E_{t}$ values are measured with APADP and thio-NADP, a result of either a nonproductive binding of the dinucleotide in the ternary E:dinucleotide:6-PG complex or a lower intrinsic $V / E_{t}$ value resulting from the change in redox potential. In agreement with these suggestions, $V$ and the $V / K$ values for 6-PG and dinucleotides decrease by the same amount when these dinucleotides are used 
TABLE I

Kinetic Parameters of Dinucleotide Substrates for 6-Phosphogluconate Dehydrogenase

\begin{tabular}{|c|c|c|c|c|c|}
\hline Dinucleotide & $V / E_{\mathrm{t}}\left(\mathrm{s}^{-1}\right)$ & $K_{\mathrm{DN}}(\mu \mathrm{M})$ & $\begin{array}{l}K_{6 \cdot \mathrm{PG}} \\
(\mu \mathrm{M})\end{array}$ & $\begin{array}{l}V / K_{\mathrm{DN}} E_{t} \\
\left(\mathrm{M}^{-1} \mathrm{~s}^{-1}\right)\end{array}$ & $\begin{array}{c}V / K_{6 \cdot \mathrm{PG}} E_{t} \\
\left(\mathrm{M}^{-1} \mathrm{~s}^{-1}\right)\end{array}$ \\
\hline$\beta$-NADP & $4.0 \pm 0.2$ & $13 \pm 2$ & $290 \pm 30$ & $(3.0 \pm 0.7) \times 10^{5}$ & $(1.4 \pm 0.1) \times 10^{4}$ \\
\hline Thio-NADP & $0.30 \pm 0.01$ & $11 \pm 1$ & $160 \pm 20$ & $(2.7 \pm 0.2) \times 10^{4}$ & $(1.8 \pm 0.2) \times 10^{3}$ \\
\hline APADP & $0.71 \pm 0.06$ & $3 \pm 1$ & $250 \pm 20$ & $(2.5 \pm 1.2) \times 10^{5}$ & $(3.0 \pm 0.6) \times 10^{3}$ \\
\hline$\epsilon-N A D P$ & $2.9 \pm 0.1$ & $32 \pm 2$ & $220 \pm 10$ & $(9.2 \pm 0.5) \times 10^{4}$ & $(1.4 \pm 0.1) \times 10^{4}$ \\
\hline HX-NADP & $4.8 \pm 0.2$ & $27 \pm 3$ & $200 \pm 30$ & $(1.5 \pm 0.1) \times 10^{5}$ & $(2.3 \pm 0.2) \times 10^{4}$ \\
\hline NAD & $0.26 \pm 0.03$ & $(16 \pm 3) \times 10^{3}$ & $71 \pm 22$ & $16.7 \pm 1.6$ & $(3.7 \pm 1.2) \times 10^{3}$ \\
\hline \multirow[t]{2}{*}{$3^{\prime}$-NADP } & $0.31 \pm 0.05$ & $(1.7 \pm 0.4) \times 10^{3}$ & & $180 \pm 25$ & \\
\hline & & & $K_{\mathrm{i}}(\mu \mathrm{M})$ & & \\
\hline$\alpha-\mathrm{NADP}$ & & & $133 \pm 11$ & & \\
\hline
\end{tabular}

${ }^{\circ}$ All assays were carried out at $\mathrm{pH} 7$ and $25^{\circ} \mathrm{C}$.

as substrates. The very low $V / K$ values measured with NAD and 3 -NADP, however, must reflect a decrease in the efficiency of the dinucleotide in triggering the conformation change needed to obtain the catalytic conformation; i.e., these dinucleotides possess a low intrinsic binding energy. Thus, 2'-phosphate containing dinucleotides likely triggers a conformational change in 6-PGDH similar to those seen for the NAD-malic enzyme (4) and for NADP-isocitrate dehydrogenase (5). The $\mathrm{p} K$ for ionization of the 2'-phosphate is not observed in the $\mathrm{pH}$ dependence of $V / K_{\mathrm{NADP}}$ or $\mathrm{p} K_{\mathrm{i} \text { ATP-ribose }}(2)$. Thus, the $2^{\prime}$ phosphate must be hydrogen-bonded to enzyme residues and can be accommodated whether or not a proton is present; i.e., the phosphate oxygens must be able to accept

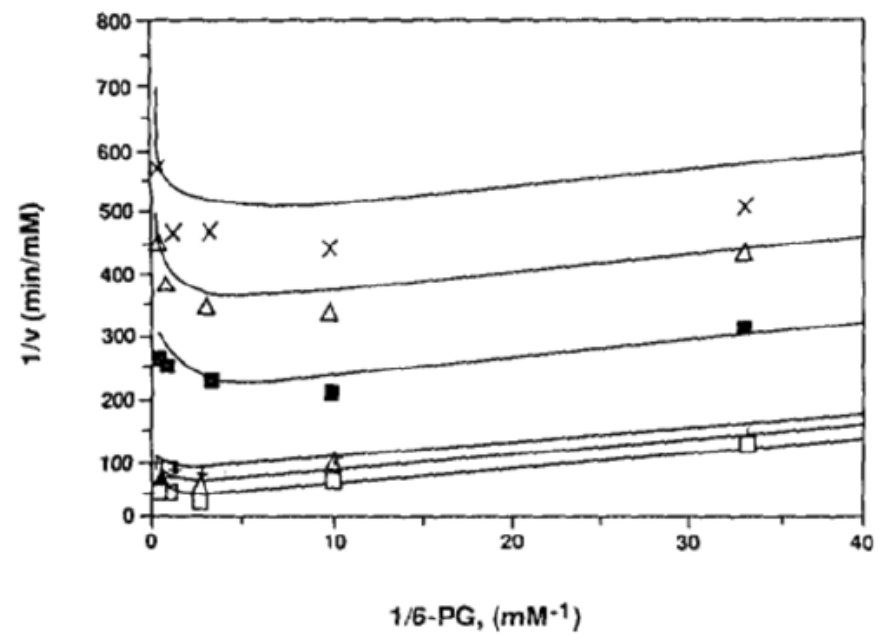

FIG. 2. Initial velocity pattern varying 6-PG at several fixed levels of NAD. Assays were carried out in $100 \mathrm{mM}$ Hepes, pH 7, with 6-PG concentrations as indicated. NAD concentrations used were (X) $1 \mathrm{~mm}$,

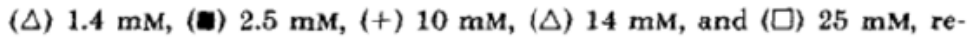
spectively. For each assay, $110 \mu \mathrm{g}$ 6-PGDH was used. The points are experimental, whereas the curves are theoretical from a fit using Eq. [3]. a hydrogen bond from an enzyme residue(s) when unprotonated and donate one to an enzyme residue(s) when protonated.

Other than the absence of a 2'-phosphate, the only other change that results in a dramatic effect is a change in the anomeric configuration at the nicotinamide ribose. If it is assumed that the enzyme is able to bind both of the bases, i.e., nicotinamide and adenine in the proper orientation, the $\alpha$-anomer would be expected to act as a substrate. Since this is not true, there must be a role for the nicotinamide ribose in the orientation of the nicotinamide ring.

The incubation of lamb liver 6-PGDH with periodateoxidized NADP causes the inactivation of the enzyme due to the covalent binding of $2 \mathrm{~mol}$ of inhibitor $/ \mathrm{mol}$ of dimeric enzyme (6). In the presence of 6-PG but not Ru 5-P or 6-SG, the inactivation is faster and is almost complete after the labeling of only one of the subunits of the dimer. Since the formation of periodate-oxidized NADPH does not occur, the acceleration should be due to a conformational change in the enzyme. These data suggest that the substrate must contain a phosphate group at carbon- 6 and a carboxyl group at carbon-1 as in 6-PG and 3-deoxy-6-PG. The binding site for 6-PG is composed of residues from the larger domain of one subunit and the carboxy-terminal of the other (7). The binding of one 6PG molecule may cause a shift of one subunit with respect to the other, possibly causing a change not only in the active site but also at the dimeric interface. If NADP acts the same as periodate-oxidized NADP, it appears that when one subunit is involved in the ternary complex (E: 6-PG:NADP) allowing the redox reaction to occur, the other subunit is unable to bind NADP, thus preventing the reaction in the second subunit. Therefore, it is likely that conformational changes occur when either substrate binds to the enzyme. The binding of 6-PG to free enzyme induces a conformational change that may allow NADP 
TABLE II

pH Dependence of Kinetic Parameters for 6-Phosphogluconate Dehydrogenase Using NAD as the Dinucleotide Substrate ${ }^{a}$

\begin{tabular}{|c|c|c|c|c|c|}
\hline $\mathrm{pH}$ & $V / E_{t}\left(\mathrm{~s}^{-1}\right)$ & $K_{\mathrm{NAD}}(\mathrm{mM})$ & $K_{6 \cdot \mathrm{PG}}(\mu \mathrm{M})$ & $\begin{array}{l}V / K_{\mathrm{NAD}} \\
\left(\mathrm{M}^{-1} \mathrm{~s}^{-1}\right)\end{array}$ & $\begin{array}{c}V / K_{6 \cdot P G} \\
\left(M^{-1} s^{-1}\right)\end{array}$ \\
\hline 7 & $0.26 \pm 0.03$ & $16 \pm 3$ & $71 \pm 22$ & $0.023 \pm 0.008$ & $4.5 \pm 1.5$ \\
\hline 8 & $11 \pm 4$ & $9 \pm 2$ & $85 \pm 33$ & $1.2 \pm 0.1$ & $134 \pm 37$ \\
\hline 9 & $22 \pm 4$ & $18 \pm 4$ & $204 \pm 88$ & $1.2 \pm 0.1$ & $108 \pm 32$ \\
\hline 9.5 & $22 \pm 3$ & $17 \pm 4$ & $640 \pm 230$ & $1.3 \pm 0.1$ & $35 \pm 8$ \\
\hline 10 & $41 \pm 14$ & $114 \pm 43$ & $(6.8 \pm 3.1) \times 10^{3}$ & $0.36 \pm 0.01$ & $6 \pm 1$ \\
\hline
\end{tabular}

${ }^{\circ}$ Data at pH 7 were fitted using Eq. [3], while data at all other pH values were fitted using Eq. [2].

to bind more effectively, while the binding of NADP to the E:6-PG complex induces the change that locks the enzyme in the catalytically competent ternary complex. In using NAD or $3^{\prime} \cdot \mathrm{NADP}$ as the dinucleotide substrate, it is possible that the binding of 6 -PG induces a conformational change as indicated above but that the binding of the dinucleotide substrate to the binary complex does not induce the conformational change to the ternary complex as well as NADP due to the lack of the $2^{\prime}$-phosphate group.

Comparison to other dehydrogenases. The pyrimidinelinked dehydrogenases of eubacterial and eukaryotic organisms are characteristically specific for either NAD or NADP, and it is uncommon to find enzymes that are equally catalytically active with both cofactors. Eukaryotes possess both NAD- and NADP-specific isocitrate de- hydrogenase. The NAD-specific enzyme (EC 1.1.1.41) is present in the mitochondria and is allosterically regulated in a manner consistent with a controlling role in the energy-yielding function of the citric acid cycle (8). The NADP-specific enzyme (EC 1.1.1.42) is present in both cytoplasm and mitochondria, but, unlike the NAD-specific enzyme, it is not subject to allosteric control (8). A variety of eubacterial species, including Acetobacter peroxydans, Xanthomonas pruni, and Hydrogenomonas eutropha, contain isocitrate dehydrogenases that use either NAD or NADP as the oxidant (9). For example, isocitrate dehydrogenase from Rhodomicrobrium vannielli has the ability to use either NAD or NADP in the oxidative decarboxylation of isocitrate. Michaelis constants for NADP and NAD are 2.5 and $770 \mu \mathrm{M}$, respectively, and values for $V / K$ using NADP and NAD are $10^{6} \mathrm{M}^{-1} \mathrm{~s}^{-1}$ and $2.5 \times 10^{3}$

TABLE III

Product and Dead-End Inhibition Patterns for 6-Phosphogluconate Dehydrogenase Using NAD as the Dinucleotide Substrate

\begin{tabular}{|c|c|c|c|c|c|c|}
\hline $\mathrm{pH}$ & Inhibitor & $\begin{array}{l}\text { Variable } \\
\text { substrate }\end{array}$ & $\begin{array}{c}\text { Fixed } \\
\text { substrate }\end{array}$ & Pattern & $K_{i \phi}(\mu \mathrm{M})$ & $K_{i i}(\mu \mathrm{M})$ \\
\hline 6 & ATP-R $R^{a}$ & NAD & 6-PG (2 mM) & $\mathrm{C}$ & $1.7 \pm 0.2$ & \\
\hline 6 & ATP-R & NAD & 6-PG (0.2 mM) & C & $1.2 \pm 0.1$ & \\
\hline 6 & ATP-R & $6 \cdot \mathrm{PG}$ & NAD (10 mM) & $\mathrm{NC}$ & $2.8 \pm 1.6$ & $4.6 \pm 0.5$ \\
\hline 6 & ATP-R & 6-PG & NAD (1 mM) & $\mathrm{NC}$ & $1.0 \pm 0.8$ & $2.5 \pm 1.0$ \\
\hline 6 & Ru 5-P & 6-PG & NAD (10 mM) & C & $1170 \pm 380$ & \\
\hline 6 & Ru 5-P & NAD & 6-PG (0.2 mM) & NC & $3100 \pm 1000$ & $1100 \pm 400$ \\
\hline 7 & ATP-R & NAD & 6-PG (3.0 mM) & $\mathrm{C}$ & $1.5 \pm 0.1$ & \\
\hline 7 & Ru 5-P & NAD & 6-PG (0.2 mM) & $\mathrm{NC}$ & $1800 \pm 400$ & $1700 \pm 800$ \\
\hline 7 & Ru 5-P & 6-PG & NAD (10 mM) & C & $\mathrm{nd}^{\mathrm{b}}$ & \\
\hline 8 & ATP-R & NAD & 6-PG (3.0 mM) & $\mathrm{C}$ & $2.5 \pm 0.4$ & \\
\hline 8 & ATP-R & 6-PG & NAD (10 mM) & $\mathrm{NC}$ & $21 \pm 12$ & $7.5 \pm 1.0(3.6)^{c}$ \\
\hline 8 & Ru 5-P & 6-PG & NAD (10 mM) & C & $600 \pm 200$ & \\
\hline 8 & Ru 5-P & NAD & 6-PG $(0.2 \mathrm{mM})$ & $\mathrm{NC}$ & $1800 \pm 350$ & $4400 \pm 1100(1300)$ \\
\hline 9 & ATP-R & NAD & 6-PG (10 mM) & $\mathrm{C}$ & $7.1 \pm 1.3$ & \\
\hline 9 & ATP-R & 6-PG & NAD (10 mM) & NC & $6.5 \pm 1.4$ & $18.1 \pm 3.5$ \\
\hline
\end{tabular}

ATP-R is ATP-ribose.

${ }^{b}$ nd, not determined, as the uninhibited line has no slope.

"Values in parentheses reflect true inhibition constants. 
TABLE IV

Structures, Redox Potentials, and Spectroscopic Data for the Dinucleotide Substrates Used in the Studies of 6-Phosphogluconate Dehydrogenase from Candida utilis ${ }^{a}$

\begin{tabular}{|c|c|c|c|c|}
\hline $\begin{array}{l}\text { Dinucleotide } \\
\text { substrate }\end{array}$ & Structure & $\begin{array}{c}\epsilon_{0}^{\prime} \\
(\mathrm{mV})\end{array}$ & $\begin{array}{c}\lambda_{\max } \\
(\mathrm{nm})^{b}\end{array}$ & $\begin{array}{l}\epsilon \text { at } \lambda_{\max } \\
\left(\mathrm{mM}^{-1}\right)^{b}\end{array}$ \\
\hline$\beta$-NADP & Fig. 2A: $\mathrm{R}_{1}=-\mathrm{PO}_{3}^{2-}, \mathrm{R}_{2}=\mathrm{H}, \mathrm{R}_{3}=-\mathrm{CONH}_{2}$ & -320 & 340 & 6.22 \\
\hline$\alpha-$ NADP $^{\mathrm{C}}$ & Fig. 2A: $\mathrm{R}_{1}=-\mathrm{PO}_{3}^{2-}, \mathrm{R}_{2}=\mathrm{H}, \mathrm{R}_{3}=-\mathrm{CONH}_{2}$ & -320 & 340 & 6.22 \\
\hline NAD & Fig. 2A: $\mathrm{R}_{1}=\mathrm{H}, \mathrm{R}_{2}=\mathrm{H}, \mathrm{R}_{3}=-\mathrm{CONH}_{2}$ & -320 & 340 & 6.22 \\
\hline $3^{\prime}$.NADP & Fig. $2 \mathrm{~A}: \mathrm{R}_{1}=\mathrm{H}, \mathrm{R}_{2}=-\mathrm{PO}_{3}^{2-},=\mathrm{R}_{3}=-\mathrm{CONH}_{2}$ & -320 & 340 & 6.22 \\
\hline$\epsilon$-NADP & Fig. 2B & -320 & 340 & 6.22 \\
\hline HX-NADP & Fig. 2C & -320 & 340 & 6.22 \\
\hline Thio-NADP & Fig. $2 \mathrm{~A}: \mathrm{R}_{1}=-\mathrm{PO}_{3}^{2-}, \mathrm{R}_{2}=\mathrm{H}, \mathrm{R}_{3}=-\mathrm{CSNH}_{2}$ & -285 & 395 & 11.30 \\
\hline APADP & Fig. $2 \mathrm{~A}: \mathrm{R}_{1}=-\mathrm{PO}_{3}^{2-}, \mathrm{R}_{2}=\mathrm{H}, \mathrm{R}_{3}=-\mathrm{COCH}_{3}$ & -258 & 363 & 9.10 \\
\hline
\end{tabular}

${ }^{a}$ Refer to Fig. 2 for the general structures of the dinucleotides.

${ }^{b}$ These values were used to follow the appearance of the corresponding reduced dinucleotide.

'Anomeric configuration at the nicotinamide ribose is opposite that shown.

$\mathrm{M}^{-1} \mathrm{~s}^{-1}$, respectively (10). Thus, it appears that dehydrogenases thought to be specific for one of the coenzymes will use the other, but with greatly reduced affinity.

The NAD:malic enzyme from Ascaris suum has a 25 fold greater affinity for NAD and a greater than 50 -fold higher $V / K$ for NAD compared to NADP (11). Comparison of the NAD or NADP binding regions of malic enzyme from A. suum, rat, murine, and human sources indicates several identical regions (12). The differences in the positions of key acidic and basic residues required to discriminate between NAD and NADP have not been definitively identified. There are significant differences at positions 364 (glutamate) and 373 (aspartate) in the two NAD-dependent enzymes ( $A$. suum and human) and in the presence of arginines at comparable positions in the NADP-dependent enzymes (rat and murine). A lysine at position 362 is interesting in the case of the ascarid enzyme since one of two basic residues, as in the murine and rat enzymes, is thought to be necessary to establish contact with the phosphate group of the NADP. The human NAD-dependent enzyme has a glutamate in this region of the protein.

The 6-PGDH Rossman fold is more similar to the NAD domain of lactate dehydrogenase than that of any other NADP enzyme of known structure (7). The Rossman fold of 6-PGDH is composed of the residues Gly-X-Ala-X$\mathrm{X}-\mathrm{Gly}$, where $\mathrm{X}$ is any amino acid. This sequence can be viewed as a hybrid of the nucleotide binding fold of NAD and NADP enzymes of known structure. The central glycine of this sequence is required due to the proximity of the adenine ribose moiety. This implies that the interaction of the dinucleotide substrate with enzyme is substantially different than any NADP-dependent enzyme of known structure. For example, in lactate dehydrogenase, aspartate 53 hydrogen bonds to the NAD ribose 2 $\mathrm{OH}$ whereas in 6-PGDH this residue is replaced by ar- ginine 33 and is most likely involved in NADP binding, interacting with the 2'-phosphate group of NADP.

The interaction of NAD and NADP with nicotinamide adenine dinucleotide-dependent enzymes has been extensively investigated by numerous techniques. Recent work has identified distinct structural motifs for NAD or NADP within the coenzyme binding region $(13,14)$ and this is thought to allow discrimination between the two coenzymes. Common to the NAD binding domains of many enzymes is a $\beta \alpha \beta$-fold centered around a highly conserved sequence, Gly-X-X-Gly-X-X-Gly (where X is any amino acid), that constitutes a tight turn at the end of the first strand of a $\beta$-sheet and marks the beginning of the succeeding $\alpha$-helix (13). The helix dipole contributes significantly to the binding of the coenzyme by interacting favorably with the pyrophosphate moeity (13). Other conserved features of this fingerprint region are (a) a hydrophobic residue at the $\mathrm{N}$-terminus of the first $\beta$ strand whose function is unknown, (b) a hydrophobic core composed of six small residues, and (c) a negatively charged residue at the $\mathrm{C}$-terminus of the second $\beta$-strand which forms a hydrogen bond with the 2 '-hydroxyl group of the adenine ribose of NAD (13). The number of tertiary structures for NADP binding domains is much smaller than that for NAD binding domains. However, in several instances the same fingerprint region can be identified, although with some significant differences. First, the negatively charged residue at the $\mathrm{C}$-terminal end of the second $\beta$-strand is replaced, presumably to accommodate the 2 'phosphate group on the coenzyme (13). Second, the third glycine residue of the conserved trio is replaced by alanine. It is important to note that some NADPH-requiring enzymes do not fit this pattern and their NADPH binding sites cannot incorporate the same compact $\beta \alpha \beta$-fold.

Kinetic mechanism using NAD as the dinucleotide substrate. Because of the unusual initial velocity pattern 
observed for the 6-PGDH reaction with NAD as a reactant (Fig. 2), the kinetics and acid/base chemistry of the reaction were studied. In the direction of the oxidative decarboxylation of 6-phosphogluconate using NAD as the dinucleotide, initial velocity patterns below $\mathrm{pH} 8$ give a series of nearly parallel lines at low 6-PG concentrations with apparent substrate inhibition by 6-PG at all concentrations of NAD (Table II). A graphical analysis of the data obtained at $\mathrm{pH} 7$ is indicative of competitive substrate inhibition by 6-PG. Data were fitted using the equation for a ping-pong kinetic mechanism with competitive substrate inhibition by $\mathrm{B}$. The $K_{\mathrm{I}}$ for 6-PG substrate inhibition is $16 \mathrm{~mm}$. All other patterns obtained below $\mathrm{pH} 8$ were qualitatively identical to that obtained at $\mathrm{pH} 7$, and quantitative data were estimated, where possible, by graphical analysis.

Since it is ludicrous to suggest a ping-pong mechanism for this reaction, it is likely that the pattern actually intersects to the left of the ordinate and below the abscissa. However, the NAD concentration range used was high with respect to $K_{\mathrm{iNAD}}$ so that the constant term $\left(K_{\mathrm{ia}} K_{\mathrm{b}}\right)$ is not observed, resulting in a set of parallel lines. Thus, a sequential kinetic mechanism is obtained with substrate inhibition by 6-PG that is apparently competitive with NAD.

Inhibition patterns were measured using $\mathrm{Ru} 5-\mathrm{P}$ and ATP-ribose as analogs of 6-PG and NAD, respectively (Table III). The product, $\mathrm{Ru} 5-\mathrm{P}$, is for all intents and purposes a dead-end analog of 6.PG since the kinetic mechanism observed with NADP as the dinucleotide substrate approximates rapid equilibrium (1) and NAD is a poorer substrate. At $\mathrm{pH} 6$ and 7, Ru 5-P is competitive vs 6-PG and noncompetitive vs NAD, while ATP-ribose is competitive vs $\mathrm{NAD}$ at any concentration of 6-PG. These data suggest a random mechanism. The mechanism observed with NADP is rapid equilibrium random (1). Since NAD is a much slower substrate with a $V / K 4 \times$ $10^{-6}$ that of NADP, the mechanism is almost certainly rapid equilibrium random with NAD. In agreement with this, the deuterium isotope effects on $V, V / K_{\mathrm{NAD}}$, and $V / K_{6 \cdot P G}$ are equal (16).

If the mechanism is still rapid equilibrium random at $\mathrm{pH} 7, K_{\mathrm{NAD}}(16 \mathrm{mM})$ reflects the dissociation constant from E:NAD:6-PG. The value of $K_{\mathrm{i} 6 \text {-PG }}$ (dissociation constant for E:6-PG), is about $200 \mu \mathrm{M}$ (1). In order to observe a nearly parallel initial velocity pattern in the case of a sequential kinetic mechanism, the lowest concentration of NAD used in these studies (1 $\mathrm{mM})$ must be at least an order of magnitude higher than the dissociation constant for E:NAD, that is $K_{\mathrm{i} \text { NAD }}$ must be less than or equal to 100 $\mu \mathrm{M}$. Using the above values and the equality $K_{\mathrm{NAD}} K_{\mathrm{i} 6 . \mathrm{PG}}$ $=K_{6 \cdot \mathrm{PG}} K_{\mathrm{iNAD}}$, which reflects the identity of the equilibrium constant for the two pathways (addition of 6-PG prior to NAD and NAD prior to 6-PG), gives a value greater than or equal to $20 \mathrm{mM}$ for $K_{6 \cdot \mathrm{PG}}$. However, a value much lower than this $(70 \mu \mathrm{M})$ is obtained from a fit using Eq. [3]. Thus, either Eq. [3] grossly underestimates $K_{6-\mathrm{PG}}$ or NAD does not bind to E:6-PG below $\mathrm{pH} 8$ for some reason; it is likely that the latter of these explanations is correct. At or above $\mathrm{pH} 8$ initial velocity patterns intersect to the left of the ordinate, indicative of a sequential mechanism, and dead-end inhibition patterns are consistent with a rapid equilibrium random kinetic mechanism. In this case, the E:NAD and E:NAD:6-PG complexes are productive.

For a rapid equilibrium random kinetic mechanism, the product $\mathrm{Ru} 5$-P behaves as a dead-end analog of the variable substrate. Quantitative analysis of the inhibition constants of Ru 5-P and ATP-ribose can be performed at $\mathrm{pH} 8$, but cannot be performed at $\mathrm{pH} 6$ and 7 due to uncertainty in the Michaelis constants obtained under these conditions or at $\mathrm{pH} 9$ since only one of the two dead-end patterns was obtained. At $\mathrm{pH} 8$, the $K_{\text {is }}$ obtained for ATP-ribose vs NAD at saturating 6-PG should be identical to the $K_{\mathrm{ii}}$ obtained for ATP-ribose vs 6-PG once corrected for the presence of NAD. The expression relating the two is $K_{\mathrm{ii}}=K_{\mathrm{i}}\left(1+\mathrm{NAD} / K_{\mathrm{NAD}}\right)$. With NAD equal to $K_{\mathrm{NAD}}$, the $K_{\mathrm{i}}$ is half the calculated $K_{\mathrm{i}}$ (given in Table III). Likewise, the $K_{\mathrm{ii}}$ obtained from the Ru 5-P vs NAD pattern can be corrected using $K_{\mathrm{ii}}=K_{\mathrm{i}}(1+6-\mathrm{PG} /$ $\left.K_{6 \cdot \mathrm{PG}}\right)$. Thus, values of 2.5 and $3.6 \mu \mathrm{M}$ for E:6-PG:ATPribose and 600 and $1300 \mu \mathrm{M}$ for E:NAD:Ru 5-P can be compared. These values are in reasonable agreement when standard errors are considered.

The kinetic mechanism is thus random with NAD as the dinucleotide substrate at all $\mathrm{pH}$ values tested. Below pH 8 there appears to be strong antagonism between 6 PG and NAD binding (Scheme I). Thus, as suggested above, the 2 '-phosphate causes a decrease in affinity of NAD for free enzyme and a more pronounced decrease in affinity and productivity of its binding to the E:6-PG complex. In agreement with this suggestion, the $\mathrm{X}$-ray structure of the E:NADP complex of 6-PGDH indicates that the tightest interaction between enzyme and NADP is at the $2^{\prime}$-phosphate (M. Adams, personal communication). The presence of the 2 '-phosphate moiety of the dinucleotide is likely required to give the proper catalytic conformation.

Predominance of one pathway in a random mechanism as the $\mathrm{pH}$ is changed is not unprecedented. Creatine ki-

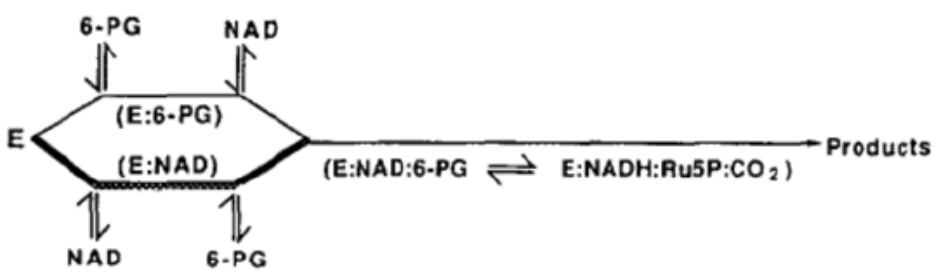

SCHEME 1. Kinetic mechanism for 6-phosphogluconate dehydrogenase from Candida utilis using NAD as the dinucleotide substrate. The boldface line represents the preferred pathway that exists below pH 8. 
nase catalyzes the reversible phosphorylation of creatine by MgATP. At pH 8, the kinetic mechanism appears to approximate rapid equilibrium random kinetics $(17,18)$. However, at $\mathrm{pH} 7$ initial velocity studies suggest an equilibrium-ordered kinetic mechanism for the phosphorylation of creatine such that MgATP binds prior to creatine (19). A reasonable explanation for this was proposed based upon the $\mathrm{pH}$ dependence of kinetic parameters using rabbit muscle creatine kinase in which the binding of creatine to give the E:creatine complex is sensitive to the protonation state of an active site histidine with a pK of $7(20)$. However, when nucleotide is bound, creatine binding is insensitive to the protonation state of the acid/base catalyst.

Studies of dopamine $\beta$-monooxygenase by Ahn and Klinman (21) have shown that the enzyme exhibits a peculiar $\mathrm{pH}$ dependence of the kinetic order for substrate release from the ternary complex such that there is a preferred order of oxygen released prior to dopamine at $\mathrm{pH}$ 4.5 , a random release at intermediate $\mathrm{pH}$ values, and a preferred order of dopamine release prior to oxygen at $\mathrm{pH}$ 6.6. There is a pH-dependent decrease in the rate of catecholamine dissociation that results in the switch of kinetic order (21).

$\mathrm{pH}$ dependence of kinetic parameters using NAD as the dinucleotide substrate. The $\mathrm{pH}$ dependence of kinetic parameters for the 6-PGDH reaction using NAD as the dinucleotide is obtained from the data in Table I. Because of the high $K_{m}$ for NAD, it is difficult to accurately measure the kinetic parameters at all $\mathrm{pH}$ values. The $V / K$ for 6-PG decreases on the basic side with a $\mathrm{p} K$ of about 8 . This value corresponds nicely to the $\mathrm{p} K$ value of 8.2 obtained using NADP as the dinucleotide and most likely reflects the general acid (2). $V_{\max }$ is $\mathrm{pH}$ independent above pH 8 but not well defined below pH 8 . These data agree reasonably well with those obtained using NADP and suggest that no dramatic changes in the acid/base chemistry occur with changes in the dinucleotide substrate.
However, the inability of NAD to bind adequately at low $\mathrm{pH}$ suggests that the protonation state of the general base with a $\mathrm{pK}$ of about 7 (2) has an effect on NAD binding, either directly or via a pH-dependent conformational change as observed for the NAD-malic enzyme (4).

\section{REFERENCES}

1. Berdis, A. J., and Cook, P. F. (1993a) Biochemistry 32, 2036.

2. Berdis, A. J., and Cook, P. F. (1993b) Biochemistry 32, 2041.

3. Cleland, W. W. (1979) Methods Enzymol. 63, 103.

4. Rajapaska, R., Abu-Soud, H., Rauschel, F. M., Harris, B. G., and Cook, P. F. (1993) Biochemistry, 32, 1928.

5. Kumosinski, T. F., Farrell, H. M., Deeney, J. T., and Hild, E. K (1990) J. Biol. Chem. 265, 17637.

6. Rippa, M., Dallocchio, F., and Hanau, S. (1992) Biochim. Biophys. Acta 1159, 262.

7. Adams, M. J., Gover, S., Leaback, R., Phillips, C., and Somers, D. O. N. (1991) Acta. Crystallogr. Sect. B 47, 817.

8. Colman, R. F. (1983) Peptide Protein Rev. 1, 41.

9. Weitzman, P. P. J. (1981) Adv. Microb. Phys. 22, 185.

10. Kelly, D. J., and Leyland, M. L. (1991) Eur. J. Biochem. 202, 85.

11. Weiss, P. M., Gavva, S. R., Harris, B. G., Urbauer, J. L., Cleland, W. W., and Cook, P. F. (1991) Biochemistry 30, 5755.

12. Kulkarni, G., Cook, P. F., and Harris, B. G. (1993) Arch. Biochem Biophys. 300, 231.

13. Hol, W. G. J., DeMaeyer, M. C. H., and Wierenga, R. K. (1985) Biochemistry 24, 1347.

14. Perham, R. N., Berry, A., and Scrutton, N. S. (1990) Nature 343, 38.

15. Schulz, G. E., Karplus, P. A., and Pai, E. F. (1988) Biochemistry 16,565 .

16. Cook, P. F. (1991) in Enzyme Mechanisms from Isotope Effects (Cook, P. F., Ed.), CRC Press, Boca Raton, FL.

17. Morrison, J. F., and Cleland, W. W. (1966) J. Biol. Chem. 241, 673.

18. Morrison, J. F., and James, E. (1965) Biochem. J. 122, 727.

19. Schimerlik, M. I., and Cleland, W. W. (1973) J. Biol. Chem. 248, 8418.

20. Cook, P. F., Kenyon, G. L., and Cleland, W. W. (1981) Biochemistry 20, 1204.

21. Ahn, N., and Klinman, J. (1983) Biochemistry 22, 3096. 